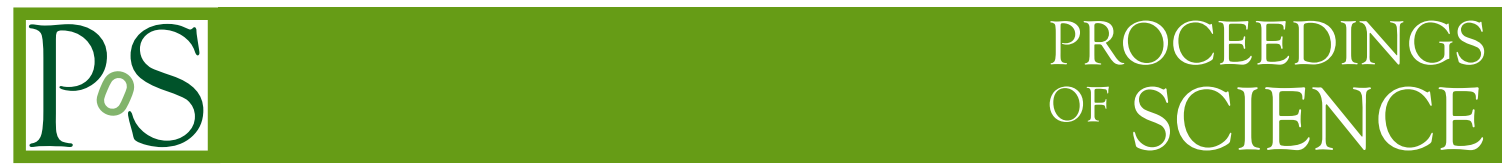

\title{
On 2D and 3D solitons in SU(2) gluodynamics
}

\author{
Alla Bogolubskaya* \\ JINR \\ E-mail: abogol@jinr.ru \\ Igor Bogolubsky \\ JINR \\ E-mail: bogolubs@jinr.ru
}

We study localized field solutions in 2D and 3D SU(2) gluodynamics. New generic 3-term ansatz is proposed for 2D case. Both in 2D and 3D Lorentz gauge is fixed, and resulting Hamiltonian densities in terms of radial functions are presented. Possible physical implications are pointed out.

35th International Conference of High Energy Physics - ICHEP2010,

July 22-28, 2010

Paris France

${ }^{*}$ Speaker. 
Study of (quasi)classical localized solutions will hopefully allow deeper insight into nonperturbative physics described by the Standard Model. Here we present some new technical results concerning stationary localized solutions of $S U(2)$ Yang-Mills field in $D=2$ and $D=3$ space dimensions with

$$
\begin{gathered}
\mathscr{L}=-\frac{1}{4}\left(F_{\mu \nu}^{a}\right)^{2}, \\
F_{\mu \nu}^{a}=\partial_{\mu} A_{v}^{a}-\partial_{v} A_{\mu}^{a}+g \varepsilon^{a b c} A_{\mu}^{b} A_{\nu}^{c}, \\
\mu, v=0,1, \ldots, D, \quad a, b, c=1,2,3,
\end{gathered}
$$

$g$ is bare coupling constant, assuming that $A_{0}^{a}=0$.

1. Consider $D=2$. We have found that the following rather generic ansatz

$$
\begin{gathered}
g A_{i k}^{a}=\delta_{a 3} \varepsilon_{i a k} x_{k} \frac{1}{R^{2}} s(R)+\left(\delta_{a 1}+\delta_{a 2}\right)\left[\left(\delta_{i a} R^{2}-x_{i} x_{a}\right) \frac{b(R)}{R^{3}}+\frac{p(R) x_{i} x_{a}}{R^{4}}\right], \\
i, k=1,2, \quad R^{2}=x^{2}+y^{2}
\end{gathered}
$$

leads to axis-symmetric energy density distributions of Hamiltonian density $\mathscr{H}_{2}(R)=$ $\mathscr{H}_{2}(s(R), b(R), p(R), R)$ :

$$
\begin{aligned}
\mathscr{H}_{2}(R)= & \frac{1}{2 g^{2}}\left[\frac{s^{2} p^{2}}{R^{2}}-\frac{b p}{R^{3}}-\frac{p}{R^{2}} \frac{d b}{d R}+\frac{1}{2}\left(\frac{d b}{d R}\right)^{2}+\frac{p^{2} s}{R^{3}}+\frac{b}{R}\left(\frac{d b}{d R}\right)+\frac{1}{2} \frac{b^{2}}{R^{2}}+\right. \\
& \left.+\frac{1}{2}\left(\frac{d s}{d R}\right)^{2}+\frac{1}{2} \frac{b^{2} p^{2}}{R^{2}}+\frac{1}{2} \frac{s^{2}}{R^{2}}+\frac{s}{R}\left(\frac{d s}{d R}\right)+\frac{b p}{R}\left(\frac{d s}{d R}\right)-\frac{s p}{R}\left(\frac{d b}{d R}\right)+\frac{1}{2} \frac{p^{2}}{R^{4}}\right] .
\end{aligned}
$$

Applying Lorentz gauge, $\partial_{\mu} A_{\mu}=0$, we arrive at energy distributions defined by 2 radial functions $b(r)$ and $s(r)$ only

$$
\mathscr{H}_{2}(R)=\frac{1}{2 g^{2}}\left\{\left[\frac{d s}{d R}+\frac{s}{R}+\frac{p}{R^{3}} \frac{d p}{d R}\right]^{2}+\frac{1}{R^{2}}\left[\frac{d^{2} p}{d R^{2}}-\frac{p}{R}\left(s+\frac{1}{R}\right)\right]^{2}\right\} .
$$

2. Now let $D=3$. We use the most generic 3-term ansatz

$$
\begin{gathered}
g A_{i}^{a}=\varepsilon_{i a k} \frac{x_{k}}{R^{2}} s(R)+\frac{b(R)}{R^{3}}\left[\left(\delta_{i a} R^{2}-x_{i} x_{a}\right)+\frac{p(R) x_{i} x_{a}}{R^{4}}\right], \\
i, k=1,2,3, \quad R^{2}=x^{2}+y^{2}+z^{2}
\end{gathered}
$$

obtaining as a result Hamiltonian density $\mathscr{H}_{3}(R)=\mathscr{H}_{3}(s(R), b(R), p(R), R)$ :

$$
\begin{aligned}
\mathscr{H}_{3}(R)= & \frac{1}{g^{2}}\left[\frac{1}{R^{2}}\left(\frac{d b}{d R}\right)^{2}+\frac{1}{R^{2}}\left(\frac{d s}{d R}\right)^{2}+\frac{2 s^{3}}{R^{4}}+\frac{2 s b^{2}}{R^{4}}-\frac{2 s p}{R^{4}}\left(\frac{d b}{d R}\right)+\frac{1}{2} \frac{b^{4}}{R^{4}}+\right. \\
& \left.+\frac{2 b p}{R^{4}}\left(\frac{d s}{d R}\right)+\frac{2 s^{2}}{R^{4}}-\frac{2 p}{R^{4}}\left(\frac{d b}{d R}\right) \frac{b^{2} s^{2}}{R^{4}}+\frac{1}{2} \frac{s^{4}}{R^{4}}+\frac{p^{2}}{R^{6}}+\frac{s^{2} p^{2}}{R^{6}}+\frac{b^{2} p^{2}}{R^{6}}+\frac{2 p^{2} s}{R^{6}}\right] .
\end{aligned}
$$

Again remove nonphysical degrees of freedom by fixing Lorentz gauge, arriving as a result at Hamiltonian density

$$
\mathscr{H}_{3}(R)=\frac{1}{g^{2}}\left\{\frac{1}{32 R^{4}}\left[\left(\frac{d p}{d R}\right)^{2}+8 s+4 s^{2}\right]^{2}+\left[\frac{p(s+1)}{R^{3}}-\frac{1}{2 R} \frac{d^{2} p}{d R^{2}}\right]^{2}+\left[\frac{1}{R} \frac{d s}{d R}+\frac{1}{2 R^{3}} \frac{d p}{d R} p\right]^{2}\right\} .
$$


3. One can see that r.h.s. of expressions (1), (2) are positively definite and contain high-order terms preventing YM lumps from collapse (in particular, 4-th order terms). Hence one can hope that 2D and 3D classical nontopological solitons of $S U(2)$ Yang-Mills fields can exist.

Presently search of radial functions $p(R)$ and $s(R)$, which correspond to local minima of energy functional $E=\int \mathscr{H}(R) R^{D-1} d R$ with $\mathscr{H}(R)$ given by (1), (2) is in progress, in particular by means of numerical lattice Monte-Carlo simulations. If such nontopological classical solitons exist, they could be used for description, e.g., interquark glounic string $(D=2)[1]$ and glueball-like states $(D=3)$.

Existence of Yang-Mills solitons was studied earlier in papers by S.Coleman et al [2-4]), where scaling transformation was used in order to prove absence of classical solitons, and corresponding "no-go theorems" have been claimed. However it is not clear for us whether spaces of variables corresponding to Yang-Mills fields, considered in these papers contain physical degrees of freedom only. It is evident that results of minimization in extended space with nonphysical degrees of freedom do not allow to obtain reliable conclusions on existence of solitons in space with extra nonphysical degrees removed, e.g. by fixing Lorentz gauge. We believe that these issues require detailed further investigation.

\section{References}

[1] G.S. Bali, K. Schilling and C. Schlichter, Observing long color flux tubes in SU(2) lattice gauge theory, PRD 51 (1995) 5165 [hep-lat/9409005].

[2] S. Coleman, Classical lumps and their quantum descendants, in proceedings of the 1975 Intern. School of Subnuclear Physics. Part A. Ed. by Zichichi (N. Y.-London: Plenum Press, 1976) 297.

[3] S. Coleman, There are no classical glueballs, Commun. Math. Phys. 55 (1977) 113.

[4] S. Deser, Absence of static EYM excitations in three dimensions, PLB 64 (1976) 463. 